\title{
A dynamic nomenclature proposal for SARS-CoV-2 lineages to assist genomic epidemiology
}

\author{
Andrew Rambaut $\mathbb{1}^{1 凶}$, Edward C. Holmes $\mathbb{1}^{2 凶}$, Áine O'Toole ${ }^{1}$, Verity Hill', John T. McCrone', \\ Christopher Ruis ${ }^{3}$, Louis du Plessis ${ }^{4}$ and Oliver G. Pybus $\mathbb{B}^{4} \bowtie$
}

The ongoing pandemic spread of a new human coronavirus, SARS-CoV-2, which is associated with severe pneumonia/disease (COVID-19), has resulted in the generation of tens of thousands of virus genome sequences. The rate of genome generation is unprecedented, yet there is currently no coherent nor accepted scheme for naming the expanding phylogenetic diversity of SARS-CoV-2. Here, we present a rational and dynamic virus nomenclature that uses a phylogenetic framework to identify those lineages that contribute most to active spread. Our system is made tractable by constraining the number and depth of hierarchical lineage labels and by flagging and delabelling virus lineages that become unobserved and hence are probably inactive. By focusing on active virus lineages and those spreading to new locations, this nomenclature will assist in tracking and understanding the patterns and determinants of the global spread of SARS-CoV-2.

\section{T} here are currently more than 35,000 publicly available complete or near-complete genome sequences of severe acute respiratory syndrome coronavirus 2 (SARS-CoV-2) (as of 1 June 2020) and the number continues to grow. This remarkable achievement has been made possible by the rapid genome sequencing and online sharing of SARS-CoV-2 genomes by public health and research teams worldwide. These genomes have the potential to provide invaluable insights into the ongoing evolution and epidemiology of the virus during the pandemic and will likely play an important role in surveillance and its eventual mitigation and control. Despite such a wealth of data, there is currently no coherent system for naming and discussing the growing number of phylogenetic lineages that comprise the population diversity of this virus, with conflicting ad hoc and informal systems of virus nomenclature in circulation. A nomenclature system for the genetic diversity of SARS-CoV-2 (a clade within the family Coronaviridae, genus Betacoronavirus, subgenus Sarbecovirus, species Severe acute respiratory syndrome-related virus ${ }^{1}$ ) is urgently required before the scientific literature and communication become further confused.

There is no universal approach to classifying virus genetic diversity below the level of a virus species ${ }^{2}$ and this is not covered by the International Committee on Taxonomy of Viruses. Typically, genetic diversity is categorized into distinct 'clades', each corresponding to a monophyletic group on a phylogenetic tree. These clades may be referred to by a variety of terms, such as 'subtypes', 'genotypes', 'groups', depending on the taxonomic level under investigation or the established scientific literature for the virus in question. The clades usually reflect an attempt to divide pathogen phylogeny and genetic diversity into a set of groupings that are approximately equally divergent, mutually exclusive and statistically well supported. Therefore, all genome sequences are allocated to one clade or provisionally labelled as 'unclassified'. Often, multiple hierarchical levels of classification exist for the same pathogens, such as the terms 'type', 'group' and 'subtype' that are used in the field of human immunodeficiency virus research.

Such classification systems are useful for discussing epidemiology and transmission when the number of taxonomic labels is roughly constant through time; this is the case for slowly evolving pathogens (for example, many bacteria) and for rapidly evolving viruses with low rates of lineage turnover (for example, human immunodeficiency virus ${ }^{3}$ and hepatitis $\mathrm{C}$ virus ${ }^{4}$ ). In contrast, some rapidly evolving viruses such as influenza $\mathrm{A}$ are characterized by high rates of lineage turnover, so that the genetic diversity circulating in any particular year largely emerges out of and replaces the diversity present in the preceding few years. For human seasonal influenza, this behaviour is the result of strong natural selection among competing lineages. In such circumstances, a more explicitly phylogenetic classification system is used. For example, avian influenza viruses are classified into 'subtypes', 'clades' and 'higher-order clades' according to several quantitative criteria ${ }^{5}$. Such a system can provide a convenient way to refer to the emergence of new (and potentially antigenically distinct) variants and is suitable for the process of selecting the component viruses for the regularly updated influenza vaccine. A similar approach to tracking antigenic diversity may be needed to inform SARS-CoV-2 vaccine design efforts. While useful, we recognize that dynamic nomenclature systems based on genetic distance thresholds have the potential to overaccumulate cumbersome lineage names.

In an ongoing and rapidly changing epidemic such as SARS-CoV-2, a nomenclature system can facilitate real-time epidemiology by providing commonly agreed labels to refer to viruses circulating in different parts of the world, thereby revealing the links between outbreaks that share similar virus genomes. Furthermore, a nomenclature system is needed to describe virus lineages that vary

IInstitute of Evolutionary Biology, University of Edinburgh, Edinburgh, UK. ${ }^{2}$ Marie Bashir Institute for Infectious Diseases and Biosecurity, School of Life and Environmental Sciences and School of Medical Sciences, University of Sydney, Sydney, New South Wales, Australia. ${ }^{3}$ Department of Medicine, University of Cambridge, Cambridge, UK. DDepartment of Zoology, University of Oxford, Oxford, UK. $\varpi_{e}$-mail: a.rambaut@ed.ac.uk; edward.holmes@sydney.edu.au; oliver.pybus@zoo.ox.ac.uk 
in phenotypic or antigenic properties. (Although it must be stressed that at present there is no conclusive evidence of such variation among currently available SARS-CoV-2 strains.)

\section{Principles of a dynamic nomenclature system}

There are several key challenges in the development of a dynamic and utilitarian nomenclature system for SARS-CoV-2. To be valid and broadly accepted a nomenclature needs to: (1) capture local and global patterns of virus genetic diversity in a timely and coherent manner; (2) track emerging lineages as they move between countries and populations within each country; (3) be sufficiently robust and flexible to accommodate new virus diversity as it is generated; and (4) be dynamic, such that it can incorporate both the birth and death of viral lineages through time.

A special challenge in the case of COVID-19 is that genome sequence data is being generated rapidly and at high volumes, such that by the end of the pandemic we can expect hundreds of thousands of SARS-CoV-2 genomes to have been sequenced. Therefore, any lineage naming system must be capable of handling tens to hundreds of thousands of virus genomes sampled longitudinally and densely through time. Furthermore, to be practical, any lineage naming system should have no more than 100 or 200 active lineage labels since any more would obfuscate rather than clarify discussion and would be difficult to conceptualize.

To fulfil these requirements, we propose a workable and practical lineage nomenclature for SARS-CoV-2 that arises from a set of fundamental evolutionary and phylogenetic principles. Some of these principles are, necessarily, specific to the COVID-19 pandemic, reflecting the new reality of large-scale real-time generation of virus genome sequences. The nomenclature system is not intended to represent every evolutionary change in SARS-CoV-2 since these will number many thousands by the end of the pandemic. Instead, the focus is on the genetic changes associated with important epidemiological and biological events. Fortunately, because of the early sampling and genome sequencing of COVID-19 cases in China, especially in Hubei province, it appears that the 'root sequence' of SARS-CoV-2 is known. Many of the genomes from the earliest sampled cases are genetically identical and hence also probably identical to the most recent common ancestor of all sampled viruses. This occurrence is different to previous viruses and epidemics and provides some advantages for the development of a rational and scalable classification scheme. Specifically, setting the 'reference sequence' to be the root sequence forms a natural starting point since direct comparisons in the number and position of mutations can be made with respect to the root sequence.

During the early phase of the pandemic, it is possible to unambiguously assign a genome to a lineage through the presence/absence of particular sets of mutations. However, a central component of a useful nomenclature system is that it focuses on those virus lineages that contribute most to global transmission and genetic diversity. Hence, rather than naming every possible new lineage, classification should focus on those that have exhibited onward spread in the population, particularly those that have seeded an epidemic in a new location. For example, the large epidemic in Lombardy, Northern Italy, thought to have begun in early February ${ }^{6}$, has since been disseminated to other locations in northern Europe and elsewhere.

Furthermore, because SARS-CoV-2 genomes are being generated continuously and at a similar pace to changes in virus transmission and epidemic control efforts, we expect to see a continual process of lineage generation and extinction through time. Rather than maintaining a cumulative list of all lineages that have existed since the start of the pandemic, it is more prudent to mark lineages as 'active,' 'unobserved' or 'inactive.' This is a designation that reflects our current understanding of whether they are actively transmitting in the population or not. Accordingly, lineages of SARS-CoV-2 documented within the last month are defined as 'active' in this article, those last seen $>1$ month but $<3$ months ago are classified as 'unobserved' and those that have not been seen for $>3$ months are termed 'inactive'.

Although this strategy allow us to track those lineages that are contributing most to the epidemic, and so reduce the number of names in use, it is important to keep open the possibility that new lineages will appear through the generation of virus genomes from unrepresented locations or from cases with travel history from such locations. For example, the epidemic in Iran (designated B.4 in our system) was identified via returning travellers to other countries? Furthermore, lineages that have not been seen for some time may re-emerge after a period of cryptic transmission in a region. Hence, it is possible for lineages that were previously classified as inactive or unobserved to be later relabelled as active. We chose the term 'lineages' (rather than 'clades', 'genotypes' or other designations) for SARS-CoV-2 because it captures the fact that they are dynamic, rather than relying on a static and exclusive hierarchical structure.

\section{Lineage naming rules}

We propose that major lineage labels begin with a letter. At the root of the phylogeny of SARS-CoV-2 are two lineages that we simply denote as lineages $\mathrm{A}$ and $\mathrm{B}$. The earliest lineage $\mathrm{A}$ viruses, such as Wuhan/WH04/2020 (EPI_ISL_406801), sampled on 5 January 2020, share two nucleotides (positions 8,782 in ORF1ab and 28,144 in ORF8) with the closest known bat viruses (RaTG13 and RmYN02). Different nucleotides are present at those sites in viruses assigned to lineage B, of which Wuhan-Hu-1 (GenBank accession no. MN908947) sampled on 26 December 2019 is an early representative. Hence, although viruses from lineage B happen to have been sequenced and published first ${ }^{8-10}$, it is likely (based on current data) that the most recent common ancestor (MRCA) of the SARS-CoV-2 phylogeny shares the same genome sequence as the early lineage A sequences (for example, Wuhan/WH04/2020). Importantly, this does not imply that the MRCA itself has been sampled and sequenced, but rather that no mutations have accrued between the MRCA and the early lineage A genome sequences. At the time of writing, viruses from both lineages A and B are still circulating in many countries around the world, reflecting the exportation of viruses from Hubei province to other regions of China and elsewhere before strict travel restrictions and quarantine measures were imposed there.

To add further lineage designations, we downloaded 27,767 complete SARS-CoV-2 genomes from the GISAID (Global Initiative on Sharing All Influenza Data) database (http://gisaid.org) ${ }^{11}$ on 18 May 2020 and estimated a maximum likelihood tree for these data (see Methods) (Fig. 1). We defined further SARS-CoV-2 lineages, each descending from either lineage $\mathrm{A}$ or $\mathrm{B}$, and assigned a numerical value (for example, lineage A.1 or lineage B.2). Lineage designations were made using the following set of conditions: (1) each descendant lineage should show phylogenetic evidence of emergence from an ancestral lineage into another geographically distinct population, implying substantial onward transmission in that population. In the case of a rapidly expanding global lineage, the recipient population may comprise multiple countries. In the case of large and populous countries, it may represent a new region or province. To show phylogenetic evidence, a new lineage must meet all of the following criteria: (a) it exhibits one or more shared nucleotide differences from the ancestral lineage; (b) it comprises at least five genomes with $>95 \%$ of the genome sequenced; (c) genomes within the lineage exhibit at least one shared nucleotide change among them; and (d) a bootstrap value $>70 \%$ for the lineage-defining node. Importantly, criterion (c) helps to focus attention only on lineages with evidence of ongoing transmission; (2) the lineages identified in step 1 can themselves act as ancestors for virus lineages that then emerge in other geographical areas or at later times, provided they satisfy criteria a-d. This results in a new lineage designation (for example, A.1.1); (3) 

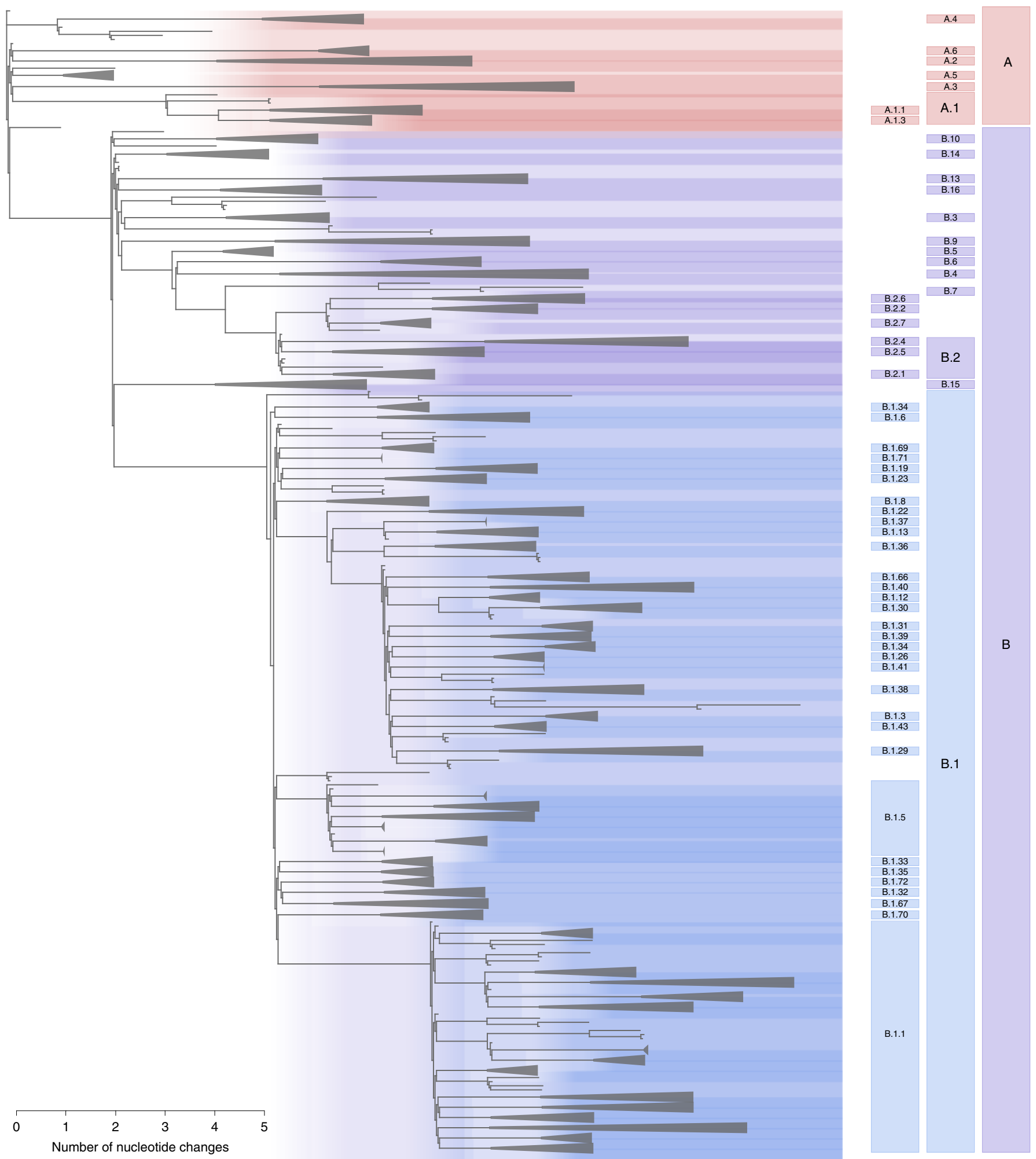

Fig. 1 | Maximum likelihood phylogeny of globally sampled sequences of SARS-CoV-2 downloaded from the GISAID database on 18 May 2020 . Five representative genomes are included from each of the defined lineages. The largest lineages defined by our proposed nomenclature system are highlighted with coloured areas and labelled on the right. The remaining lineages defined by the nomenclature system are denoted by triangles. The scale bar represents the number of nucleotide changes within the coding region of the genome.

the iterative procedure in step 2 can proceed for a maximum of three sublevels (for example, A.1.1.1) after which new descendant lineages are given a letter (in English alphabetical sequence from C) so A.1.1.1.1 would become C.1 and A.1.1.1.2 would become C.2.
The rationale for this is that the system is intended only for tracking currently circulating lineages, such that we do not try to capture the entire history of a lineage in its label. (That complete history can be obtained by reference to a phylogeny.) At the time of writing, 


\begin{tabular}{|c|c|c|c|}
\hline Lineage & Genomes & Date range & Comments \\
\hline A & 223 & $\begin{array}{l}5 \text { January-27 } \\
\text { April } 2020\end{array}$ & $\begin{array}{l}\text { The root of the pandemic lies } \\
\text { in this lineage. Many Chinese } \\
\text { sequences with global exports }\end{array}$ \\
\hline A.1 & 1,116 & $\begin{array}{l}20 \text { February-25 } \\
\text { March } 2020\end{array}$ & $\begin{array}{l}\text { Primary outbreak in } \\
\text { Washington State, USA }\end{array}$ \\
\hline A. 2 & 295 & $\begin{array}{l}26 \text { February-27 } \\
\text { April } 2020\end{array}$ & European lineage \\
\hline A. 3 & 191 & $\begin{array}{l}28 \text { January-21 } \\
\text { April } 2020\end{array}$ & USA lineage \\
\hline A. 5 & 118 & $\begin{array}{l}23 \text { February-26 } \\
\text { April } 2020\end{array}$ & European lineage \\
\hline B & 1,713 & $\begin{array}{l}24 \text { December } \\
2019-3 \text { May } \\
2020\end{array}$ & $\begin{array}{l}\text { The base of this lineage lies in } \\
\text { China, with extensive global } \\
\text { travel between multiple } \\
\text { locations }\end{array}$ \\
\hline B.1 & 7,438 & $\begin{array}{l}24 \text { January-10 } \\
\text { May } 2020\end{array}$ & $\begin{array}{l}\text { Comprises the large Italian } \\
\text { outbreak; it now represents } \\
\text { many European outbreaks, } \\
\text { with travel within Europe and } \\
\text { from Europe to the rest of the } \\
\text { world }\end{array}$ \\
\hline B.1.1 & 6,286 & $\begin{array}{l}15 \text { February-9 } \\
\text { May } 2020\end{array}$ & $\begin{array}{l}\text { Major European lineage; } \\
\text { exports to the rest of the world } \\
\text { from Europe }\end{array}$ \\
\hline B.2 & 917 & $\begin{array}{l}13 \text { February-4 } \\
\text { May } 2020\end{array}$ & $\begin{array}{l}\text { With B.1, it comprises the large } \\
\text { Italian outbreak }\end{array}$ \\
\hline B.3 & 752 & $\begin{array}{l}23 \text { February-23 } \\
\text { April } 2020\end{array}$ & UK lineage \\
\hline B.4 & 258 & $\begin{array}{l}18 \text { January-14 } \\
\text { April } 2020\end{array}$ & $\begin{array}{l}\text { This is probably the primary } \\
\text { Iranian outbreak }\end{array}$ \\
\hline
\end{tabular}

See https://cov-lineages.org/ for full details of each lineage.

no C level lineages have been assigned; (4) all sequences are assigned to one lineage. For example, if a genome does not meet the criteria for inclusion in a 'higher-level' lineage (for example, A.1.2, B.1.3.5) then it is automatically classified into the lowest level for which it meets the inclusion criteria, which ultimately is A or B.

Using this scheme, we identified 81 viral lineages. These lineages mostly belong to A, B and B.1. We identified six lineages derived from lineage A (denoted A.1-A.6) and two descendant sublineages of A.1 (A.1.1 and A.3). We also describe 16 lineages directly derived from lineage B. To date, lineage B.1 is the predominant known global lineage and has been subdivided into $>70$ sublineages. Lineage B. 2 currently has six descendant sublineages. We are not yet able to further subdivide the other lineages, even though some contain very large numbers of genomes. This is because many parts of the world experienced numerous imported cases followed by exponential growth in local transmission. We provide descriptions of these initial lineages, including their geographical locations and time span of sampling, in Table 1 . We have also tried to be flexible with the criteria where, for example, the bootstrap value is below $70 \%$ but there is strong previous evidence that the lineage exists and is epidemiologically important. In particular, the Italian epidemic comprises two large lineages in our scheme-B.1 and B.2-reflecting genomes from Italy as well as from large numbers of travellers from these regions and that fall into both lineages.

A unique and important aspect of our proposed nomenclature is that the status of the currently circulating lineages be assessed at regular intervals, with decisions made about identifying new lineages and flagging those we believe are likely be unobserved or inactive because none of their members have been sequenced for a considerable time. The names of unobserved or inactive lineages will not be reassigned. These are provisional timescales and the category thresholds may be altered in the future once the dynamics of lineage generation and extinction are better understood. When visualizing the epidemic, we suggest that these lineages should no longer be labelled to reduce both the number of names in circulation and visual noise, and focus on the current epidemiological situation.

\section{Discussion}

While we regard this proposed nomenclature as practical and robust, it is important to recognize that phylogenetic inference carries statistical uncertainty and much of the available genome data is noisy, with incomplete genome coverage and errors arising from the amplification and sequencing processes. We have suggested a genome coverage threshold for proposing new lineages and we further suggest that sequences are not ascribed a lineage designation unless the genome coverage of that sequence exceeds $70 \%$ of the coding region. As noted earlier, when SARS-CoV-2 genetic diversity is low during the early pandemic period, there is a direct association between lineage assignation and the presence of particular sets of mutations (with respect to the root sequence). This should help with the development of rapid, algorithmic genome labelling tools. This task will become more complex, but still tractable, as SARS-CoV-2 genetic diversity accumulates, increasing the chance of both homoplasies and reverse mutations. Classification algorithms based on lists of 'lineage-defining' mutations may be practical if they are frequently cross-checked and validated against phylogenetic estimations but will not be as powerful as phylogenetic classification methods, which make use of complete genome sequence data to identify relationships. We encourage the research community to develop software and online tools that will enable the automated classification of newly generated genomes (one such implementation is pangolin, https://github.com/hCoV-2019/pangolin).

Coronaviruses also frequently recombine, meaning that a single phylogenetic tree may not always adequately capture the evolutionary history of SARS-CoV-2. Although this can make phylogenetic analysis challenging, recombination is readily accommodated within this system of lineage naming and assignment. A distinct recombination event, if it establishes onward transmission, will create a new viral lineage with a distinct common ancestor. Because this new lineage does not have a single ancestral lineage, it will be assigned the next available alphabetical prefix.

While we believe our proposed lineage nomenclature will greatly assist those working with COVID-19, we do not see it as exclusive to other naming systems, particularly those that are specifically intended to track lineages circulating within individual countries for which a finer scale will be helpful. Indeed, there are likely to be strong sampling biases towards particular countries. Furthermore, we note that future genome sequence generation may require adjustments to the current proposal; any such changes will be detailed at http:// cov-lineages.org/. However, we envisage that the general approach described in this study may be readily adopted for these purposes and for other viral epidemics where real-time genomic epidemiology is being undertaken. We expect this dynamic nomenclature to be most useful for the duration of the global pandemic, which may last a few years. After that time, SARS-CoV-2 will be either globally eliminated or, more likely, become an endemic or seasonal infection. The remaining endemic/seasonal lineages, which will be genetically distinct by then, can simply retain their names from the dynamic nomenclature system in the post-pandemic period.

\section{Methods}

We downloaded all SARS-CoV-2 genomes (at least 29,000 base pairs in length) from GISAID on 18 May 2020 . We trimmed the $5^{\prime}$ - and $3^{\prime}$-untranslated regions 
and retained those genomes with at least $95 \%$ coverage of the reference genome (Wuhan-Hu-1). We aligned these sequences using the MAFFT FFT-NS-2 algorithm and default parameter settings ${ }^{12}$. We then estimated a maximum likelihood tree using IQ-TREE 2 ( ref. ${ }^{13}$ ) using the GTR $+\Gamma$ model of nucleotide substitution $^{14,15}$, default heuristic search options and ultrafast bootstrapping with 1,000 replicates $^{16}$

The maximum likelihood tree and associated sequence metadata were manually curated and the phylogeny was annotated with the lineage designations. This annotated tree, along with a table providing the lineage designation for each genome in the dataset, is available for download at http://cov-lineages.org/. We have also provided a high-resolution figure (in PDF format) of the entire tree labelled with lineages. These will be updated on a regular basis. Representative sequences from each lineage were selected to maximize within-lineage diversity and minimize $\mathrm{N}$-content and used to construct the maximum likelihood tree shown in Fig. 1.

Reporting Summary. Further information on research design is available in the Nature Research Reporting Summary linked to this article.

\section{Data availability}

No new data have been reported. The viral genome sequences used in this paper are publicly available from GISAID (http://gisaid.org). A table of acknowledgements for the GISAID genome sequences used to develop this work is available at https://cov-lineages.org/gisaid_acknowledgements.

\section{Code availability}

Details of the software and source code that implement the nomenclature system reported in this paper are available at http://cov-lineages.org.

Received: 16 April 2020; Accepted: 3 July 2020;

Published online: 15 July 2020

\section{References}

1. Walker, P. J. et al. Changes to virus taxonomy and the international code of virus classification and nomenclature ratified by the international committee on taxonomy of viruses (2019). Arch. Virol. 164, 2417-2429 (2019).

2. ICTV Code: the International Code of Virus Classification and Nomenclature International Committee on Taxonomy of Viruses (ICTV) https://talk.ictvonline. org/information/w/ictv-information/383/ictv-code/ (2018).

3. Robertson, D. L. et al. HIV-1 nomenclature proposal. Science 288, 55-56 (2000)

4. Smith, D. B. et al. Expanded classification of hepatitis $C$ virus into 7 genotypes and 67 subtypes: updated criteria and genotype assignment web resource. Hepatology 59, 318-327 (2014).

5. WHO/OIE/FAO H5N1 Evolution Working Group. Continued evolution of highly pathogenic avian influenza A (H5N1): updated nomenclature. Influenza Other Respir. Viruses 6, 1-5 (2012).

6. Zehender, G. et al. Genomic characterization and phylogenetic analysis of SARS-CoV-2 in Italy. J. Med. Virol. https://doi.org/10.1002/jmv.25794 (2020)

7. Eden, J.-S. et al. An emergent clade of SARS-CoV-2 linked to returned travellers from Iran. Virus Evol. 6, veaa027 (2020).

8. Lu, R. et al. Genomic characterisation and epidemiology of 2019 novel coronavirus: implications for virus origins and receptor binding. Lancet 395 565-574 (2020).
9. $\mathrm{Wu}, \mathrm{F}$. et al. A new coronavirus associated with human respiratory disease in China. Nature 579, 265-269 (2020).

10. Zhu, N. et al. A novel coronavirus from patients with pneumonia in China, 2019. N. Engl. J. Med. 382, 727-733 (2020).

11. Shu, Y. \& McCauley, J. GISAID: Global Initiative on Sharing All Influenza Data-from vision to reality. Euro Surveill. 22, 30494 (2017).

12. Katoh, K., Misawa, K., Kuma, K.-I. \& Miyata, T. MAFFT: a novel method for rapid multiple sequence alignment based on fast Fourier transform. Nucleic Acids Res. 30, 3059-3066 (2002).

13. Minh, B. Q. et al. IQ-TREE 2: new models and efficient methods for phylogenetic inference in the genomic era. Mol. Biol. Evol. 37, 1530-1534 (2020).

14. Tavaré, S. Some probabilistic and statistical problems in the analysis of DNA sequences. In Proc. of the Symposium on Some Mathematical Questions in Biology: DNA Sequence Analysis. Lectures on Mathematics in the Life Sciences (ed. Miura, R. M.) 57-86 (American Mathematical Society, 1986).

15. Yang, Z. Maximum likelihood phylogenetic estimation from DNA sequences with variable rates over sites: approximate methods. J. Mol. Evol. 39, 306-314 (1994).

16. Minh, B. Q., Nguyen, M. A. T. \& von Haeseler, A. Ultrafast approximation for phylogenetic bootstrap. Mol. Biol. Evol. 30, 1188-1195 (2013).

\section{Acknowledgements}

This work was funded by the Wellcome Trust Collaborative Award (grant no. 206298/Z/17/Z-ARTIC Network), the European Research Council under the European Union's Horizon 2020 research and innovation programme (grant no. 725422-ReservoirDOCS), the European Commission Seventh Framework Programme (grant no. FP7/2007-2013)/European Research Council (grant no. 614725-PATHPHYLODYN), the UK COVID-19 Genomics Consortium, the Oxford Martin School and the Australian Research Council (grant no. FL170100022).

\section{Author contributions}

A.R., E.C.H. and O.G.P. conceived, designed and supervised the study. Á.O.T., J.T.M. and A.R. developed the phylogenetic methods. A.R., Á.O.T., V.H., J.T.M., C.R., L.D.P. and O.G.P. analysed and interpreted the viral genomes. A.R., E.C.H., A.O.T., V.H. and O.G.P. wrote the paper with input from the other authors.

\section{Competing interests}

The authors declare no competing interests.

\section{Additional information}

Supplementary information is available for this paper at https://doi.org/10.1038/ s41564-020-0770-5.

Correspondence and requests for materials should be addressed to A.R., E.C.H. or O.G.P.

Peer review information Peer reviewer reports are available.

Reprints and permissions information is available at www.nature.com/reprints.

Publisher's note Springer Nature remains neutral with regard to jurisdictional claims in published maps and institutional affiliations.

(c) The Author(s), under exclusive licence to Springer Nature Limited 2020 


\section{Reporting Summary}

Nature Research wishes to improve the reproducibility of the work that we publish. This form provides structure for consistency and transparency in reporting. For further information on Nature Research policies, see Authors \& Referees and the Editorial Policy Checklist.

\section{Statistics}

For all statistical analyses, confirm that the following items are present in the figure legend, table legend, main text, or Methods section.

n/a Confirmed

$\triangle \square$ The exact sample size $(n)$ for each experimental group/condition, given as a discrete number and unit of measurement

\ $\square$ A statement on whether measurements were taken from distinct samples or whether the same sample was measured repeatedly

$\searrow$ The statistical test(s) used AND whether they are one- or two-sided

$\triangle$ Only common tests should be described solely by name; describe more complex techniques in the Methods section.

Х $\square$ A description of all covariates tested

Х $\square$ A description of any assumptions or corrections, such as tests of normality and adjustment for multiple comparisons

Х A full description of the statistical parameters including central tendency (e.g. means) or other basic estimates (e.g. regression coefficient)

Х $\square$ AND variation (e.g. standard deviation) or associated estimates of uncertainty (e.g. confidence intervals)

$\triangle$ For null hypothesis testing, the test statistic (e.g. $F, t, r$ ) with confidence intervals, effect sizes, degrees of freedom and $P$ value noted

$\triangle$ Give P values as exact values whenever suitable.

$\bigotimes \square$ For Bayesian analysis, information on the choice of priors and Markov chain Monte Carlo settings

\ $\square$ For hierarchical and complex designs, identification of the appropriate level for tests and full reporting of outcomes

$\bigotimes \square$ Estimates of effect sizes (e.g. Cohen's $d$, Pearson's $r$ ), indicating how they were calculated

Our web collection on statistics for biologists contains articles on many of the points above.

\section{Software and code}

Policy information about availability of computer code

Data collection No software used for data collection.

Data analysis Custom code available at http://cov-lineages.org

Analysis used open source software: MAFFT v7.458, IQTree v1.6.12, Biopython v1.74, Dendropy v4.4.0

For manuscripts utilizing custom algorithms or software that are central to the research but not yet described in published literature, software must be made available to editors/reviewers. We strongly encourage code deposition in a community repository (e.g. GitHub). See the Nature Research guidelines for submitting code \& software for further information.

\section{Data}

Policy information about availability of data

All manuscripts must include a data availability statement. This statement should provide the following information, where applicable:

- Accession codes, unique identifiers, or web links for publicly available datasets

- A list of figures that have associated raw data

- A description of any restrictions on data availability

This study uses data from the GISAID SARS-CoV-2 repository (http://gisaid.org/). An acknowledgment table is available on the Github repository: http://covlineages.org/gisaid_acknowledgements.html 


\section{Field-specific reporting}

Please select the one below that is the best fit for your research. If you are not sure, read the appropriate sections before making your selection. \Life sciences Behavioural \& social sciences Ecological, evolutionary \& environmental sciences

For a reference copy of the document with all sections, see nature.com/documents/nr-reporting-summary-flat.pdf

\section{Life sciences study design}

All studies must disclose on these points even when the disclosure is negative.

Sample size The study used 27,767 SARS-CoV-2 publicly available genome sequences.

Data exclusions Genome sequence data was excluded if it was of insufficient coverage or quality to produce a reliable phylogenetic placement.

Replication Replication is not applicable to this study.

Randomization Randomization is not applicable to this study.

Blinding Blinding is not applicable to this study.

\section{Reporting for specific materials, systems and methods}

We require information from authors about some types of materials, experimental systems and methods used in many studies. Here, indicate whether each material, system or method listed is relevant to your study. If you are not sure if a list item applies to your research, read the appropriate section before selecting a response.

Materials \& experimental systems Methods

Involved in the study
$\square$ Antibodies
$\square$ Eukaryotic cell lines
$\square$ Palaeontology
$\square$ Clinical data

$\mathrm{n} / \mathrm{a}$ Involved in the study

Х $\square$ ChIP-seq

Х $\square$ Flow cytometry

Х) $\square$ MRI-based neuroimaging 